\title{
Cytomegalovirus: an unrecognised potential contributor to cystic fibrosis disease progression?
}

\author{
To the Editor:
}

Cytomegalovirus (CMV) is a betaherpesvirus, the impacts of which are well known to clinicians providing post-transplant cystic fibrosis care. Lung transplant recipients have the highest risk of any solid-organ transplant for CMV reactivation and ganciclovir resistance $[1,2]$. Furthermore, CMV reactivation increases the risk of chronic lung allograft dysfunction. However, even in general populations, CMV seropositivity is associated with adverse outcomes including cognitive impairment, frailty, heart disease and all-cause mortality [3-5]. How CMV may contribute to disease is not evident but many streams of evidence suggest CMV replication in inflamed sites contributes to exaggerated inflammation and tissue injury [6]. Individuals with cystic fibrosis experience chronic inflammation within the airways leading to remodelling and eventually respiratory failure. Indeed, inflammatory biomarkers in the sputum and serum of cystic fibrosis patients correlate with short- and long-term outcomes [7]. We hypothesised that CMV may represent an unrecognised contributor to cystic fibrosis lung disease.

We performed a detailed chart review of all Calgary Adult Cystic Fibrosis Clinic patients who were referred for lung transplantation, where CMV IgG testing would be performed. CMV serostatus, demographics, infecting pathogens, markers of nutrition and lung function from last clinical encounter were recorded for those who were transplanted or succumbed to disease. Our primary outcome was a composite end-point of age at lung transplantation/death without transplant. We also analysed the outcomes of death and lung transplant separately by CMV serostatus, and conducted a stratified analysis per time period to account for improvements in care (1990-1999, 2000-2009 and 2010-2017). Ethical approval for this study was supplied from the Conjoint Health Region Ethics Board of the University of Calgary (REB15-2744).

Sociodemographic and clinical characteristics, including referral and listing for transplantation, were summarised. Univariate and multivariable linear regression models were constructed for the primary outcome to compare $\mathrm{CMV}^{+}$and $\mathrm{CMV}^{-}$patients. We incorporated clinical (disease severity and microbiology) factors that varied significantly between patients by CMV status as well as sex, body mass index (BMI) and educational status (less than high school, high school, technical college or university) (which trended toward significance and were biologically plausible). Nonparametric tests of trend were conducted to compare outcomes between periods. Significance was based on $\alpha<0.05$, all hypothesis tests were two-sided and statistical analysis was performed using Stata version 14.2 (StataCorp, College Station, TX, USA).

Since 1991, 71 patients (50.7\% female) were referred and listed for transplant consideration who either successfully received a lifesaving transplantation or died waiting. Of these, 59 (83\%) received bilateral lung transplants and $12(16.9 \%)$ died prior to transplantation. Of the cohort, 15 patients were excluded (six died and nine transplanted) from the analysis as they did not have CMV serology documented. Patients who were excluded were demographically similar but were more likely to represent earlier cohorts $(44 \%$ in $1990-1999,14 \%$ in 2000-2009 and 0\% in 2010-2017; p=0.004).

Of the 56 included, 30 (54.6\%) were $\mathrm{CMV}^{+}$and the prevalence of CMV seropositivity did not differ by time period. When sociodemographic and clinical characteristics were examined, patients who were $\mathrm{CMV}^{-}$

@ERSpublications

Of cystic fibrosis (CF) patients referred for lung transplant consideration, those who are cytomegalovirus $(\mathrm{CMV}) \mathrm{IgG}^{+}$at referral are 8 years younger than seronegatives, suggesting $\mathrm{CMV}$ may have a pathogenic role in CF lung disease http://ow.ly/iSQf30nAxxl

Cite this article as: Parkins MD, Ramos KJ, Goss $\mathrm{CH}$, et al. Cytomegalovirus: an unrecognised potential contributor to cystic fibrosis disease progression? Eur Respir J 2019; 53: 1801727 [https://doi.org/10.1183/ 13993003.01727-2018]. 
were more likely to be F508del homozygous and have Burkholderia cepacia complex infection (all Burkholderia multivorans) but otherwise did not differ from $\mathrm{CMV}^{+}$individuals (table 1). Lung transplantation occurred a median of 1.25 years (interquartile range (IQR) $0.87-1.78$ years) after referral and 0.44 years (IQR 0.14-0.90 years) following listing, and did not differ by CMV status.

For the primary outcome, patients who were $\mathrm{CMV}^{+}$died or underwent bilateral lung transplantation at a significantly younger age compared to those who were $\mathrm{CMV}^{-}$(27.17 versus 35.11 years; difference 7.95 years (95\% CI 3.61-12.29 years), $\mathrm{p}<0.001$ ). The difference in age at death or lung transplant remained significant in a multivariable model adjusted for sex, BMI, B. cepacia complex infection, genotype and education (6.96 years (95\% CI 2.51-11.4 years), $\mathrm{p}=0.003$ ). When the outcomes of death and transplant were assessed separately, $\mathrm{CMV}^{+}$was associated with a significantly lower mean age at either death (difference 9.35 years (95\% CI $0.89-17.82$ years), $\mathrm{p}=0.03$ ) or lung transplantation (difference 7.36 years (95\% CI 2.58-12.13 years), $\mathrm{p}=0.003$ ). No significant differences in age at death or transplant were noted by CMV serostatus $(\mathrm{p}>0.05)$ when outcomes were stratified by time periods.

Our retrospective study demonstrated a significant age disparity at death or lung transplantation in persons with cystic fibrosis, suggesting a deleterious association for CMV. However, we must consider several limitations to these observations. Most importantly, association does not confirm causation [8]. This was at a single-centre study with a limited sample size and limited power to detect potentially meaningful differences in the outcome in the stratified analyses. Although there was no evidence of changing rates of CMV seroprevalence, the study spanned $\geqslant 25$ years and considerable improvements in cystic fibrosis outcomes have since been realised. However, when we analysed our outcomes stratified by time periods, no significant changes in the association were observed. A portion of patients were excluded from analysis due to missing CMV status leading to potential selection bias but they were demographically similar to the analysed cohort. While we were able to identify CMV serostatus of patients at the time of transplant

TABLE 1 Characteristics of cohort at time of referral for lung transplantation consideration

\begin{tabular}{lcc} 
Factor & $\mathbf{C M V}^{-}$ & $\mathbf{C M V}^{+}$ \\
\hline Patients & 26 & 30 \\
Female & $12(46 \%)$ & $17(57 \%)$ \\
F508 homozygous & $19(76 \%)$ & $13(43 \%)$ \\
$\geqslant 1$ F508 allele & $23(92 \%)$ & $21(70 \%)$ \\
Pancreatic insufficiency & $24(96 \%)$ & $27(90 \%)$ \\
CF-related diabetes & $6(24 \%)$ & $10(33 \%)$ \\
CF liver disease & $6(24 \%)$ & $4(13 \%)$ \\
CF arthropathy & $5(20 \%)$ & $4(13 \%)$ \\
CF sinus disease & $12(48 \%)$ & $15(50 \%)$ \\
Recurrent DIOS & $9(36 \%)$ & $14(47 \%)$ \\
GORD & $17(68 \%)$ & $18(60 \%)$ \\
FEV1 \% predicted & $21(18-27)$ & $25(21-30)$ \\
FVC $\%$ predicted & $50.5(42-58)$ & $44.5(38-49)$ \\
BMI kg-m ${ }^{-2}$ & $20.5(18.5-22.0)$ & $19.7(17.6-20.8)$ \\
Receipt of enteral nutrition & $14(58 \%)$ & $12(40 \%)$ \\
Supplemental oxygen & $24(96 \%)$ & $28(93 \%)$ \\
Oxygen L-min & -1 & $4(3-5)$ \\
Pseudomonas aeruginosa & $3(3-4)$ & $28(93 \%)$ \\
BCC & $22(88 \%)$ & $1(3 \%)$ \\
Stenotrophomonas maltophilia & $6(24 \%)$ & $3(10 \%)$ \\
MSSA & $2(8 \%)$ & $8(27 \%)$ \\
MRSA & $7(28 \%)$ & 0 \\
Achromobacter spp. & $1(4 \%)$ & $1(3 \%)$ \\
Aspergillus & 0 & $5(17 \%)$ \\
Did not complete high school & $6(24 \%)$ & $9(30 \%)$ \\
High school as highest degree & $5(20 \%)$ & $12(40 \%)$ \\
College or technical diploma & $7(28 \%)$ & $2(7 \%)$ \\
University & $6(24 \%)$ & $7(23 \%)$ \\
\hline & $7(28 \%)$ & \\
\hline
\end{tabular}

Data are presented as median (interquartile range) unless otherwise stated. CMV: cytomegalovirus; CF: cystic fibrosis; DIOS: distal intestinal obstruction syndrome; GORD: gastro-oesophageal reflux diseases; FEV1: forced expiratory volume in $1 \mathrm{~s}$; FVC: forced vital capacity; BMI: body mass index; BCC: Burkholderia cepacia complex; MSSA: methicillin-susceptible Staphylococcus aureus; MRSA: methicillin-resistant S. aureus. 
referral, we were unable to determine when patients were infected and infection duration may have differential effects on clinical outcomes. Within general populations, CMV seropositivity increases over time with rates increasing from $36 \%$ in those $6-11$ years, $49 \%$ in those $20-29$ years, $65 \%$ at $40-49$ years and $>91 \%$ in those $>80$ years of age [9]. Accordingly, duration of CMV infection may be a more important predictor of progression to the primary outcome as opposed to serostatus at a single time-point. Furthermore, quantitative levels of CMV IgG may be more sensitive at identifying risk as opposed to current reporting of qualitative results [4]. CMV seropositivity is known to exist disproportionally in socially marginalised groups [10]; socioeconomic status is an important modifier of cystic fibrosis [11], and influences access to lung transplant referral and listing [12]. However, we did not identify significant differences between groups in socioeconomic status using education indicators.

Our knowledge of CMV pathogenesis offers a potential model through which CMV may accelerate disease progression in cystic fibrosis: 1) the lungs are a major site of CMV reactivation [13], and progressive lung disease is responsible for the majority of CF morbidity and mortality; 2) CMV reactivation can be triggered by bacterial infection [14] and bacterial infection is omnipresent in cystic fibrosis; 3) CMV reactivation is associated with reduced immune surveillance [15] and thus, increased susceptibility to infections; and 4) CMV reactivation is associated with an exaggerated immune response [16], potentially enhancing tissue damage. CMV infection could partially explain the varied clinical courses experienced by individuals with cystic fibrosis, and why conventional microbiological markers correlate poorly with pulmonary exacerbation recovery and long-term outcomes [17].

To confirm whether CMV associates with disease progression in cystic fibrosis, we can leverage CMV serostatus data collected as part of transplant databases (e.g. the International Society for Heart and Lung Transplantation Registry) in efforts to understand CMV pathogenesis pre- and post-transplantation, i.e. to confirm the results of this single-centre study. Furthermore, cross-sectional seroprevalence studies of general cystic fibrosis populations captured with national registries will enable us to correlate critical markers of cystic fibrosis disease progression with CMV status such as baseline lung function, changes in lung function and exacerbation frequency. We would advocate for prospective longitudinal studies of cystic fibrosis populations to assess factors associated with incident infections and seroconversion, in order to determine whether acquisition of CMV infection is associated with change in disease status and how infection duration correlates with clinical outcomes. Indeed, a case series of three individuals experiencing exacerbations in the context of CMV seroconversion was recently published [18]. Finally, exploring the role of CMV reactivation/viraemia and its association with exacerbation occurrence and recovery and rates of lung function decline is important, as demonstrated by efforts currently underway to explore CMV pathogenesis in intensive care units [19].

The identification of the association of CMV with cystic fibrosis lung disease progression could have tremendous implications for disease management. If this association is confirmed in larger multicentre studies, strategies exploring the use of pre-emptive and prophylactic antiviral treatments, including acyclovir (which while not having significant CMV activity, can reduce reactivation potential) or valganciclvoir, in specific populations may be in order [20]. Considerable progress in developing an effective vaccine for $\mathrm{CMV}$ has been made recently and a large number of products are currently being investigated [21]. The rationale for these vaccines has predominately focused on their role in preventing congenital cases of CMV and avoiding transplant complications in pre-transplant patients. However, it may be that individuals with cystic fibrosis who are seronegative for CMV may derive benefit from effective vaccine strategies both acutely and in the long-term setting of their risk for transplant.

Michael D. Parkins ${ }^{1}$, Kathleen J. Ramos ${ }^{2}$, Christopher H. Goss ${ }^{2}$ and Ranjani Somayaji ${ }^{1}$

${ }^{1}$ Dept of Medicine, University of Calgary, Calgary, AB, Canada. ${ }^{2}$ Dept of Medicine, University of Washington, Seattle, WA, USA.

Correspondence: Michael D. Parkins, Dept of Medicine, University of Calgary, 3330 Hospital Drive, NW, Calgary, Alberta, T2N 4N1, Canada. E-mail: mdparkin@ucalgary.ca

Received: Sept 142018 | Accepted after revision: Jan 102019

Author's contributions: M.D. Parkins was primarily responsible for the data collection and record maintenance. Statistical analyses were performed by R. Somayaji and M.D. Parkins. M.D. Parkins was responsible for the creation of the initial draft of the manuscript. All authors contributed to development of the final manuscript. M.D. Parkins is the guarantor of this work.

Conflict of interest: M.D. Parkins reports grants from CF Canada, CIHR Canada, Gilead Sciences and the Alberta and NWT Lung Association, outside the submitted work. K.J. Ramos reports grants from the National Institutes of Health, Cystic Fibrosis Foundation and CHEST Foundation, outside the submitted work. C.H. Goss reports grants from the Cystic Fibrosis Foundation, European Commission and NIH (NHLBI, NIDDK and NCRR), during the conduct of the study; and personal fees from Gilead Sciences and Novartis, grants from the NIH and FDA, and serving as the US lead 
in a phase 2 trial of a novel therapy for cystic fibrosis for Boehringer Ingelheim, outside the submitted work.

R. Somayaji reports grants from CF Canada, CIHR Canada and Alberta Innovates, outside the submitted work.

\section{References}

1 Razonable RR, Humar A. Cytomegalovirus in solid organ transplantation. Am J Transplant 2013; 13: Suppl. 4, 93-106.

2 Thomas LD, Milstone AP, Miller GG, et al. Long-term outcomes of cytomegalovirus infection and disease after lung or heart-lung transplantation with a delayed ganciclovir regimen. Clin Transplant 2009; 23: 476-483.

3 Simanek AM, Dowd JB, Pawelec G, et al. Seropositivity to cytomegalovirus, inflammation, all-cause and cardiovascular disease-related mortality in the United States. PLoS One 2011; 6: e16103.

4 Roberts ET, Haan MN, Dowd JB, et al. Cytomegalovirus antibody levels, inflammation, and mortality among elderly Latinos over 9 years of follow-up. Am J Epidemiol 2010; 172: 363-371.

5 Barnes LL, Capuano AW, Aiello AE, et al. Cytomegalovirus infection and risk of Alzheimer disease in older black and white individuals. J Infect Dis 2015; 211: 230-237.

6 Compton T, Kurt-Jones EA, Boehme KW, et al. Human cytomegalovirus activates inflammatory cytokine responses via CD14 and Toll-like receptor 2. J Virol 2003; 77: 4588-4596.

7 Liou TG, Adler FR, Keogh RH, et al. Sputum biomarkers and the prediction of clinical outcomes in patients with cystic fibrosis. PLoS One 2012; 7: e42748.

8 Lederer DJ, Bell SC, Branson RD, et al. Control of confounding and reporting of results in causal inference studies. Guidance for authors from editors of respiratory, sleep, and critical care journals. Ann Am Thorac Soc 2019; 16: 22-28.

9 Staras SA, Dollard SC, Radford KW, et al. Seroprevalence of cytomegalovirus infection in the United States, 1988-1994. Clin Infect Dis 2006; 43: 1143-1151.

10 Dowd JB, Aiello AE, Alley DE. Socioeconomic disparities in the seroprevalence of cytomegalovirus infection in the US population: NHANES III. Epidemiol Infect 2009; 137: 58-65.

11 O'Connor GT, Quinton HB, Kneeland T, et al. Median household income and mortality rate in cystic fibrosis. Pediatrics 2003; 111: e333-e339.

12 Quon BS, Psoter K, Mayer-Hamblett N, et al. Disparities in access to lung transplantation for patients with cystic fibrosis by socioeconomic status. Am J Respir Crit Care Med 2012; 186: 1008-1013.

13 Cook $\mathrm{CH}$, Zhang Y, Sedmak DD, et al. Pulmonary cytomegalovirus reactivation causes pathology in immunocompetent mice. Crit Care Med 2006; 34: 842-849.

14 Cook $\mathrm{CH}$, Zhang $\mathrm{Y}$, McGuinness $\mathrm{BJ}$, et al. Intra-abdominal bacterial infection reactivates latent pulmonary cytomegalovirus in immunocompetent mice. J Infect Dis 2002; 185: 1395-1400.

15 Boeckh M, Nichols WG. Immunosuppressive effects of beta-herpesviruses. Herpes 2003; 10: 12-16.

16 Mansfield S, Griessl M, Gutknecht M, et al. Sepsis and cytomegalovirus: foes or conspirators? Med Microbiol Immunol 2015; 204: 431-437.

17 Parkins MD, Rendall JC, Elborn JS. Incidence and risk factors for pulmonary exacerbation treatment failures in patients with cystic fibrosis chronically infected with Pseudomonas aeruginosa. Chest 2012; 141: 485-493.

18 Sawant A, Spoletini G, Whitaker P, et al. Cytomegalovirus-associated pulmonary exacerbation in patients with cystic fibrosis. ERJ Open Res 2018; 4: 00111-2017.

19 Limaye AP, Stapleton RD, Peng L, et al. Effect of ganciclovir on IL-6 Levels among cytomegalovirus-seropositive adults with critical illness: a randomized clinical trial. JAMA 2017; 318: 731-740.

20 Barkholt L, Lewensohn-Fuchs I, Ericzon BG, et al. High-dose acyclovir prophylaxis reduces cytomegalovirus disease in liver transplant patients. Transpl Infect Dis 1999: 1: 89-97.

21 Schleiss MR. Cytomegalovirus vaccines under clinical development. J Virus Erad 2016; 2: 198-207. 